\title{
Overconfidence and market efficiency with heterogeneous agents*
}

\author{
Diego García $\quad$ Francesco Sangiorgi ${ }^{\ddagger} \quad$ Branko Urošević $^{\S}$
}

October 13, 2004

\begin{abstract}
We study financial markets in which both rational and overconfident agents coexist and make endogenous information acquisition decisions. We demonstrate the following irrelevance result: when a positive fraction of rational agents (endogeneously) decides to become informed in equilibrium, prices are set as if all investors were rational, and as a consequence the overconfidence bias does not affect informational efficiency, price volatility, rational traders' expected profits or their welfare. Intuitively, as overconfidence goes up, so does price infornativeness, which makes rational agents cut their information acquisition activities, effectively undoing the standard effect of more aggressive trading by the overconfident.
\end{abstract}

JEL classification: D80, G10.

Keywords: partially revealing equilibria, overconfidence, rational expectations, information acquisition, price informativeness.

\footnotetext{
${ }^{*}$ We would like to thank Alberto Bisin, Xavier Freixas, Ken French, Moshe Kim, Jose Marín, and Terrance Odean for comments on an early draft, as well as seminar participants at HEC Geneva, the 2004 EFA meetings, and the 2004 European Econometric Society meetings. All remaining errors are our own. The latest version of this paper can be downloaded from http://diego-garcia.dartmouth.edu.

${ }^{\dagger}$ Correspondence information: Diego García, Tuck School of Business Administration, Hanover NH 037559000, US, tel: 1-603-646-3615, fax: 1-603-646-1308, email: diego.garcia@Dartmouth.edu. Also affiliated with SECCF (Belgrade).

${ }^{\ddagger}$ Francesco Sangiorgi, Department of Economics and Business, Universitat Pompeu Fabra, Barcelona, Spain, and LUISS Guido Carli, Rome. Email: francesco.sangiorgi@upf.edu.

${ }^{\S}$ Branko Urošević, Department of Economics and Business, Universitat Pompeu Fabra, 08005, Barcelona, Spain, tel: 34-93-542-2590, email: branko.urosevic@upf.edu. Also affiliated with CREA (Barcelona) and SECCF (Belgrade).
} 


\section{Introduction}

Bounded rationality of economic agents participating in financial markets has been a subject of intense scrutiny in the last decade (see, for example, Thaler (1992), Thaler (1993), and Shleifer (2000)). One such well-documented behavioral pattern is investor overconfidence. ${ }^{1}$ Our paper contributes to the emerging literature on the effects of behavioral biases in financial markets by studying the reaction of rational agents to the degree of overconfidence of a set of irrational traders. To the best of our knowledge, this is the first paper that simultaneously adopts two important features or real financial markets: 1) coexistence of rational and overconfident traders, ${ }^{2}$ and 2 ) endogenous information acquisition by agents. In particular, we extend the existing literature by analyzing the impact that the presence of heterogenous (i.e. rational and overconfident) traders has on informational efficiency of prices, willingness of agents to acquire information, market liquidity, and performance and welfare of rational (and overconfident) agents.

Most of the existing models with overconfidence assume exogenous distribution of information among the economic agents. Such simplification is not innocuous: since traders' overconfidence impacts the market precisely through the incorrect interpretation of their private signals on the fundamental value of the traded asset, the effects of overconfidence in the economy may crucially depend on the distribution of information among the agents. It seems natural, therefore, not to specify a priori the information that different agents possess, but to instead allow it to arise endogenously. We first show that overconfidence will reduce rational agents' incentives to gather information within the standard competitive rational expectations paradigm (Grossman and Stiglitz (1980), Hellwig (1980)). We further show that a simple condition on the primitives of the model exists under which the process of competitive trading itself is a mechanism able to prevent overconfidence from affecting several important characteristics of competitive equilibrium, such as informational efficiency and volatility of asset prices, as well as welfare and the expected profits of rational agents. In fact, none of these properties are affected by the presence of overconfident traders in the economy (and coincide with the values in the purely rational economy), if the degree of overconfidence in the economy is below a certain threshold.

To gain intuition for this result note that overconfident, by overestimating the precision of

\footnotetext{
${ }^{1}$ For an excellent review on psychological literature on overconfidence see Odean (1998) and references therein. For empirical evidence on overconfidence in financial markets see, also, Barber and Odean (2001), Glaser and Weber (2003), and Statman, Thorley, and Vorkink (2003), among many others.

${ }^{2}$ Long, Shleifer, Summers, and Waldmann (1990), DeLong, Shleifer, Summers, and Waldmann (1991), Shleifer and Vishny (1997), and Bernardo and Welch (2001), among others, demonstrate that irrational traders may have long-term viability and can coexist with rational traders. Gervais and Odean (2001) find that initially over-confident traders tend to, over time, become more rational so that, cross-sectionally, at any given point in time a fraction of overconfident traders cohabitates with their rational colleagues.
} 
their signal, trade more aggressively on their private signals than rational traders. In doing so, more information is revealed by the price. Rational agents react to such anticipated behavior of the overconfident by scaling down their own demand for information, aiming to neutralize the negative externality imposed by overconfidence on the rational agents' expected profits and welfare. This "reaction" can be observed only when rational traders are free to decide whether or not to become informed. Thus, endogeneity of information acquisition is crucial for this result to hold. By the same token, and somewhat surprisingly, presence of overconfidence in a fraction of trader population leads to a decrease in a fraction of informed population as opposed to an increase (as argued elsewhere in the literature). On the other hand, overconfidence will always matter for other market statistics such as trading volume. In particular, trading volume always increases in overconfidence, a result well established theoretically as well as empirically (see Barber and Odean (2001), for example).

Several recent theoretical studies focus on the effects of overconfidence on key features of financial markets, as well as on the performance of overconfident traders. ${ }^{3}$ Kyle and Wang (1997), Odean (1998) and Benos (1998) consider models with informed insiders and noise traders submitting market orders and find that overconfidence generally leads to an increase in trading volume, market depth and price informativeness. Both Kyle and Wang (1997) and Benos (1998) allow for rational agents in their models, but information acquisition decisions are fixed in both models. ${ }^{4}$ Odean (1998), heuristically, argues that the introduction of rational traders to his model "would mitigate but not eliminate the effects of overconfident traders". 5 Rubinstein (2001) summarizes the effects of overconfidence by stating that "[overconfidence] does create a positive externality for passive investors who now find that prices embed more information and markets are deeper than they should be." We show that precisely due to this externality, rational agents will reduce their information gathering activities, and that indeed, under fairly general conditions, this can eliminate the standard positive effect of overconfidence on price informativeness.

The paper is organized as follows. Section 2 presents a competitive model with endogenous information acquisition. The irrelevance result is developed in detail in section 3. Section 4 considers various extensions, where we argue that the results discussed in the paper are robust to the types of financial market model we consider in the main body of the paper. Section 5 concludes and outlines possible directions of future research. Proofs are relegated to the Appendix.

\footnotetext{
${ }^{3}$ See Caballé and Sàkovics (2003), Daniel, Hirshleifer, and Subrahmanyam (1998), Daniel, Hirshleifer, and Subrahmanyam (2001), and Scheinkman and Xiong (2004) for some recent work.

${ }^{4}$ In Model III, Odean (1998) allows traders can decide to purchase a single piece of costly information. The author finds that in an economy with only overconfident traders, a greater degree of overconfidence leads to a larger fraction of traders that would decide to become informed in equilibrium. In contrast to our paper, Odean (1998) does not model rational traders.

${ }^{5}$ See Odean (1998), Model I.
} 


\section{The model}

The basic model in this paper extends the standard one period rational expectations model with endogenous information acquisition (see Hellwig (1980) and Verrechia (1982)) to the setting in which overconfident (irrational) economic agents coexist with rational ones. In particular, we assume that a measure $m_{o} \in(0,1)$ of the trader population is of the type $o$ (overconfident), while the measure $m_{r} \in(0,1)$ is of the type $r$ (rational). All traders in the economy have CARA preferences with risk aversion parameter $\tau$, i.e. their utility function, defined over the terminal wealth, is $u\left(W_{i}\right)=-e^{-\tau W_{i}}$. There are two assets in the economy: a riskless asset (the numeraire) in perfectly elastic supply (its gross return is, without loss of generality, normalized to 1 ), and a risky asset with payoff $X$ and random supply $Z$. Letting $\theta_{i}$ denote the number of units of the risky asset bought by agent $i$, and letting $P_{x}$ denote its price, we have that the final wealth of a trader $i$ is given by $W_{i}=\theta_{i}\left(X-P_{x}\right)^{6}$.

Each trader can decide to purchase a noisy signal about the payoff of the risky asset, which we will denote by $Y_{i}=X+\epsilon_{i}$, at a cost $c>0$. Therefore, for an uninformed trader's $i$ information set $\mathcal{F}_{i}$ consists of the risky asset price $P_{x}$ while for the informed the information set contains, also, the signal. ${ }^{7}$ All random variables $X, Z$ and $\epsilon_{i}$ are independent Gaussian random variables, defined on a probability space $(\Omega, \mathcal{F}, \mathbb{P})$, with zero mean and variances equal, respectively, to $\sigma_{x}^{2}, \sigma_{z}^{2}$ and $\sigma_{\epsilon}^{2}$. We further normalize the payoff of the risky asset $X$ so that $\sigma_{x}^{2}=1$.

In the basic setup, the only difference between the two types of traders is that type $o$ traders incorrectly believe that the variance of the signal $\sigma_{\epsilon}^{2}$ is equal to $k_{\epsilon} \sigma_{\epsilon}^{2}$, where $k_{\epsilon} \in(0,1)$. Thus, traders of type $o$ overestimate the precision of the signal. We will also parametrize overconfidence by $b_{\epsilon}=k_{\epsilon}^{-1}$, so that the higher values of $b_{\epsilon}$ are associated with higher degrees of overconfidence. In contrast, traders of type $r$ correctly estimate the precision of the signal (for such traders $b_{\epsilon}=1$ ). Type $j=o, r$ expectations are denoted as $\mathbb{E}^{j}$. Here, agents of type $r$ compute the expectations vis-a-vis the true measure (we denote $\mathbb{E}^{r}$ as $\mathbb{E}$ for brevity), while the agents of the type $o$, those with a behavioral bias, compute their expectations, denoted by $\mathbb{E}^{o}$, using the probability measure that underestimates the variance of the signal (i.e. that uses $k_{\epsilon} \sigma_{\epsilon}^{2}$ instead of $\sigma_{\epsilon}^{2}$ ).

Every trader in the economy is a price-taker and knows the structure of the market. In particular, each type $j=o, r$ knows that the other type has different beliefs about the precision of the signal. ${ }^{8}$ The timing in the model is as follows. For each type $j=o, r$, a fraction $\lambda_{j}$ of

\footnotetext{
${ }^{6}$ Notice that we normalized initial wealth to zero.

${ }^{7}$ Formally, we will denote an informed agent's information set by $\mathcal{F}_{I}$ (the $\sigma$-algebra generated by $\left(Y_{i}, P_{x}\right)$ ) and an uninformed agent's information set by $\mathcal{F}_{U}$ (the $\sigma$-algebra corresponding to the risky asset price $P_{x}$ ).

${ }^{8}$ In equilibrium, traders properly deduce the fraction of the population of each trader type that, in equilibrium, becomes informed. Thus, we do not consider a situation such as the one studied in Morrison (2004) where
} 
the respective population decides to acquire a signal. Once that decision is made, each trader submits the demand schedule for the risky asset conditional on her information set $\left(\mathcal{F}_{I}\right.$ or $\left.\mathcal{F}_{U}\right)$. The price is set to clear the market. Finally, the fundamental value of the risky asset is revealed and the endowments consumed.

Let us assume that some exogenously given fractions $\lambda_{j}(j=o, r)$ of the agents decide to become informed. The next definition is standard.

Definition 1 An equilibrium in the economy is defined by a set of trading strategies $\theta_{i}$ and a price function $P_{x}: \Omega \rightarrow \mathbb{R}$ such that:

1. Each agent $i$ of type $j$ chooses her trading strategy so as to maximize her expected utility given her information set $\mathcal{F}_{i}$ :

$$
\theta_{i} \in \arg \max _{\theta} \mathbb{E}^{j}\left[u\left(W_{i}\right) \mid \mathcal{F}_{i}\right]
$$

2. The market clears:

$$
m_{o} \Theta_{o}+m_{r} \Theta_{r}=Z
$$

where $\Theta_{j}=\frac{1}{m_{j}} \int_{0}^{m_{j}} \theta_{i} d i$ is the per capita (average) trade by the type $j$ agents $(j=o, r)$.

For the equilibrium with endogenous information acquisition to be well defined, we need to introduce two basic assumptions regarding the information technology.

Definition 2 We call an information technology non-trivial if $C(\tau)^{-1} b_{\epsilon}>\sigma_{\epsilon}^{2}$, where $C(\tau) \equiv$ $e^{2 c \tau}-1$.

Definition 3 We say that the information technology satisfies the no free lunch condition if $\Lambda^{*}<1$, where

$$
\Lambda^{*}=\frac{1}{m_{r}}\left(\tau \sigma_{\epsilon} \sigma_{z} \sqrt{C(\tau)^{-1}-\sigma_{\epsilon}^{2}}-m_{o} b_{\epsilon}\right)
$$

The first assumption requires that the information technology has a sufficiently high priceto-quality ratio so that some traders find it optimal to invest in information acquisition activities. If the condition did not hold no agent would ever become informed in equilibrium. The second assumption is a bit more restrictive: it limits our study to economies where not all agents decide to purchase information. ${ }^{9}$ In the rest of the paper, we assume that the informa-

the fraction of informed traders is unknown to traders at the moment of trading.

${ }^{9}$ If one thinks of $c$ as the price that a profit maximizing seller of information would charge for the signal, such seller of information will never choose $c$ that would violate $\Lambda^{*} \leq 1$ (for technical simplicity we use a strong 
tion technology is non-trivial and does not allow free lunch. The setup thus far closely parallels Diamond (1985), which is a special variation of the model discussed in Verrechia (1982). ${ }^{10}$

\section{Equilibrium prices}

This section solves for the competitive equilibrium with information acquisition, and derives main results of the paper including the irrelevance result.

\subsection{The competitive equilibrium with information acquisition}

As is customary in models with endogenous information acquisition, the model is solved in two stages: we first determine the equilibrium asset price function by taking $\lambda_{j}$ as exogenously fixed; then we go back to the information acquisition stage and find the equilibrium values for $\lambda_{j}$, thus completing the specification of equilibrium.

Lemma 1 For given values of $\lambda_{j} \geq 0$, the competitive equilibrium price $P_{x}$ is given by the expression $P_{x}=\hat{a} X-\hat{d} Z$, where the coefficients $\hat{a}$ and $\hat{d}$ satisfy:

$$
\begin{gathered}
\frac{\hat{a}}{\hat{d}} \equiv \gamma=\frac{1}{\tau \sigma_{\epsilon}^{2}}\left(\lambda_{o} m_{o} b_{\epsilon}+\lambda_{r} m_{r}\right) ; \\
\hat{d}=\frac{1+\frac{\gamma}{\tau \sigma_{z}^{2}}}{\gamma+\frac{\gamma^{2}}{\tau \sigma_{z}^{2}}+\frac{1}{\tau}} .
\end{gathered}
$$

The informational contents of price, or simply the market efficiency, is measured by the conditional variance of the fundamental asset value, given the market price. From Lemma 1 it follows that this quantity is given by:

$$
\operatorname{var}\left(X \mid P_{x}\right)=\left(1+\frac{\gamma^{2}}{\sigma_{z}^{2}}\right)^{-1} .
$$

The smaller the conditional variance (6), the more information is revealed by the price in equilibrium. Since the information revealed by the price monotonically increases in $\gamma$,

inequality in the definition, ruling out the knife-edge case $\Lambda^{*}=1$ ). Indeed, it can be seen from the definition of the equilibrium in the next section that doing so would mean that such seller of information would charge less for the information than the price at which every agent in the economy would become informed. Thus, such seller of information would be "leaving money on the table."

${ }^{10}$ The main difference from those models is that we relax their assumption that there are only rational agents in the economy. 
comparative statics of $\gamma$ encapsulates everything we need to know about the dependence of (6) on the parameters measuring the overconfidence in the economy. It is straightforward to see that, when $\lambda_{j}$ are fixed:

$$
\frac{d \gamma}{d b_{\epsilon}}=\frac{m_{o} \lambda_{o}}{\tau \sigma_{\epsilon}^{2}} \geq 0
$$

From (7) it follows that, when $\lambda_{o}$ is exogenous and positive, an increase in the intensity of overconfidence $b_{\epsilon}$ raises the amount of information revealed by the price. The intuition for this result is the same as in Odean (1998), namely, the more overconfident traders are, the more aggressively they trade on their information, which makes the price more informative.

The next Lemma characterizes the equilibrium with endogenous information acquisition.

Lemma 2 The equilibrium with information acquisition belongs to one of the following two classes:

(a) If the parameters of the model are such that $\Lambda^{*}>0$, a fraction of the rational agents and all overconfident agents become informed, i.e. $\lambda_{o}^{*}=1$ and $\lambda_{r}^{*}=\Lambda^{*}$.

(b) If the parameters of the model are such that $\Lambda^{*} \leq 0$, a fraction of the overconfident traders becomes informed and no rational trader becomes informed, i.e. $\lambda_{r}^{*}=0$ and

$$
\lambda_{o}^{*}=\frac{\tau \sigma_{z}}{m_{o}} \sqrt{k_{\epsilon} \sigma_{\epsilon}^{2}\left(C(\tau)^{-1}-k_{\epsilon} \sigma_{\epsilon}^{2}\right)}
$$

The above Lemma shows that depending on the values of the primitives that characterize the economy, different types of equilibria may endogenously arise: traders who decide to acquire the signal and become informed can be either only a fraction of overconfident traders, all overconfident but no rational traders, all overconfident and a fraction of rational traders, or all traders in the economy. The relevant property of the equilibrium is that rational traders become informed only if all overconfident traders are informed. This result is intuitive since overconfident overestimate the precision of the signal, and therefore it cannot be that some rational trader decides to become informed and an overconfident does not. ${ }^{11}$

It is straightforward to show that fixing other parameter values, region $\Lambda^{*}>0$ arises when: (i) degree of overconfidence $m_{o} b_{\epsilon}$ is sufficiently small; (ii) information acquisition costs $c$ are sufficiently low and/or the variability of the aggregate supply shock $\sigma_{z}$ is large; (iii) values of the risk-aversion $\tau$ and signal precision $\sigma_{\epsilon}^{2}$ are intermediate. The first two conditions are rather intuitive: if there are many overconfident agents, or their bias is too high, they will

\footnotetext{
${ }^{11}$ In the existing literature with overconfidence and asymmetric information, it is typically argued that those traders that do not buy the information are those that value it properly (see, for instance, Odean (1998), page 1907 and Daniel, Hirshleifer, and Subrahmanyam (2001), page 928). Our results confirm this assertion.
} 
crowd out the rational agents, and we are back to the setting where the overconfident are the marginal buyers of information. If the cost is low or the noise large, traders find information acquisition activities more attractive, eventually making the rational traders (marginal) buyers of information. The third result comes from the dual role that those two parameters, riskaversion and signal precision, play in this type of competitive models. On one hand they affect the value of becoming informed: more risk-tolerant agents are willing to pay more for a signal, and more precise signals are more valuable to agents. At the same time these parameter values affect the information revealed by prices: more risk-tolerant agents, or agents with more precise signals, trade more aggressively thereby exacerbating the negative externality of their trades. It can be shown that this second effect dominates for small values of $\tau$ and $\sigma_{\epsilon}^{2}$, which pushes down the fraction of informed agents towards zero. At the same time, as both $\tau$ and $\sigma_{\epsilon}^{2}$ grow without bound agents eventually have no incentives to buy information, and again we do not satisfy the $\Lambda^{*}>0$ condition.

\subsection{Irrelevance result and comparative statics}

In the following Proposition we state the main irrelevance result on overconfidence.

Proposition 1 If $\Lambda^{*}>0$ then overconfidence is irrelevant for informational efficiency, the parameters of the equilibrium price function, unconditional variance and rational traders expected returns and welfare, that is, these quantities are equal to those that would endogenously arise in a fully rational economy.

We can interpret $\Lambda^{*}=0$ as an irrelevance threshold and think of this result in the following way. Compare two economies characterized by a common set of primitives (variances and risk aversion): one in which $m_{o}=0$ (fully rational economy) and one in which $m_{o}>0$, i.e., in which a positive measure of overconfident traders interacts with rational traders. The above Proposition states that as long as $\Lambda^{*}>0$ (or, equivalently, $m_{o} b_{\epsilon}$ is below the threshold value $\left.\tau \sigma_{\epsilon} \sigma_{z} \sqrt{C(\tau)^{-1}-\sigma_{\epsilon}^{2}}\right)$ the two economies will have identical asset prices.

The intuition for this result lies in the reaction on the part of rational traders to the presence of overconfidence. From the equilibrium equation for $\gamma$ in (4), we have that for $\Lambda^{*}>0$

$$
\frac{d \gamma}{d b_{\epsilon}}=\frac{1}{\tau \sigma_{\epsilon}^{2}}\left(m_{o}+m_{r} \frac{d \lambda_{r}^{*}}{d b_{\epsilon}}\right)
$$

The first term, $m_{o} / \tau \sigma_{\epsilon}^{2}$, is the standard term stemming from more aggressive trading by the overconfident agents as $b_{\epsilon}$ increases. The second term, which measures the (negative) reaction of the rational population to the increase in overconfidence, is what drives the irrelevance result. A simple inspection of (3), and noting that $\lambda_{r}^{*}=\Lambda^{*}$, yields that $\gamma$ is indeed independent of 
the overconfidence parameter $b_{\epsilon} \cdot{ }^{12}$ In turn, this implies that the parameters of the equilibrium price function (see equations (4) and (5)) do not depend on overconfidence parameters and are given by the same quantities as in the fully rational economy. ${ }^{13}$ As a consequence, the same is true for the unconditional variance, expected utilities and the expected returns of the rational traders.

To gain some further intuition for this result suppose that one starts from a point in which $m_{o}=0$ and $\lambda_{r}^{*} \in(0,1)$ and consider the effect that overconfident traders would have upon entering the market, i.e. what happens if $m_{o}$ becomes positive. These new traders acquire information with probability one (since some rational traders are already informed) and they value the signal $Y_{i}$ higher than their rational counterparts due to their behavioral bias. The fact that they overvalue the precision of their signals makes them trade more aggressively than what rational informed traders would do in their place. If the equilibrium fraction of informed rational traders $\lambda_{r}^{*}$ were to remain constant, this would result in higher informational efficiency (higher value of $\gamma$ ), as discussed after Lemma 1. But, since the previous level of $\gamma$ was optimal, rational traders react by adjusting $\lambda_{r}^{*}$ that diminishes so as to keep $\gamma$ constant. Overconfident traders are revealing more information through their trades, increasing the incentive to the rational agents to free ride on the public information that can be extracted from the price. The fraction of informed agents $\lambda_{r}^{*}$ diminishes so as to keep the expected utilities of rational informed and uninformed equal, and it does so because acquiring costly information is a strategic substitute. ${ }^{14}$

While previous studies argue that overconfidence is costly to society (see, for instance, Odean (1998)), Proposition 1 gives the conditions under which the process of competitive trading itself is a mechanism able to prevent overconfidence from affecting the informational efficiency of the price, and the welfare and profits of the rational traders. In this case overconfidence can be costly only to the overconfident.

On the other hand, as long as $\Lambda^{*}>0$ is satisfied, the two economies (the fully rational and the one with overconfidence) will exhibit some interesting differences, described in the next Proposition.

Proposition 2 If $\Lambda^{*}>0$ then: (i) the measure of informed traders is lower that what would be observed in a fully rational economy, (ii) overconfident traders earn higher expected returns than

\footnotetext{
${ }^{12}$ Similarly, differentiating (3) with respect to $m_{o}$ one can see that $\gamma$ does not depend on $m_{o}$ either.

${ }^{13}$ In essence, this result can be generated in an economy with agents with two different risk-aversion parameters, say $\bar{\tau}>\underline{\tau}$. If the high risk-aversion agents are the marginal buyers of information, then changes in the risk-aversion parameter $\underline{\tau}$ will not affect price informativeness. Therefore, these results can be viewed as a precise statements under which the weak inequalities in Verrechia (1982), in terms of the effects of risk-aversion on price informativeness, hold as equalities.

${ }^{14}$ For a rational expectations model in which learning can be a strategic complement, see Barlevy and Veronesi (2000).
} 
rational traders, and (iii) expected trading volume is increasing in parameters of overconfidence.

We will discuss these three results in order. Result (i) is surprising. In fact, it goes in the opposite direction of what previous literature finds: Odean (1998), for example, considers a model where overconfident traders can decide to acquire a single piece of information, and finds that too many of them are willing to buy it. We find that the measure of informed traders, both rational and overconfident, is lower than in the corresponding rational economy. This is rather intuitive: when $m_{o}$ or $b_{\epsilon}$ increases, $\gamma$ remains constant, but since the overconfident reveal more of their signal than rational traders, now a smaller measure of informed is sufficient to sustain a given level of $\gamma$.

The second result stems from the behavioral bias of overconfident traders: the more overconfident traders are the higher risks they are willing to take, which yields higher expected returns, although the risk-return trade-off they achieve is worse (using the true probability measure) than that of the rational agents. Result (iii) on trading volume confirms the robustness of previous findings on the effect of overconfidence on the expected trading volume (e.g. Odean (1998)). Namely, increase in the degree of overconfidence $m_{o} b_{\epsilon}$ enhances the expected trading volume by affecting the trading aggressiveness of the measure of informed overconfident.

So far we discussed the situation in which the degree of overconfidence is below the threshold. When that is not the case, i.e. when the degree of overconfidence exceeds the irrelevance threshold, an increase in overconfidence affects the economy in the way described by the following Proposition.

Proposition 3 If the parameters of the model are such that $\Lambda^{*} \leq 0$, then: (i) increase in overconfidence raises informational efficiency, (ii) expected returns and welfare are not increasing in overconfidence, (iii) the unconditional price volatility is non-monotonic in overconfidence, and (iv) trading volume is increasing in overconfidence.

Above the irrelevance threshold, ${ }^{15}$ only a fraction of overconfident and no rational traders become informed in equilibrium. Going back to the expression for $\gamma$, which measures price informativeness, we see that in that case:

$$
\frac{d \gamma^{*}}{d b_{\epsilon}}=\frac{1}{\tau \sigma_{\epsilon}^{2}}\left(m_{o} \frac{d\left(\lambda_{o}^{*} b_{\epsilon}\right)}{d b_{\epsilon}}\right)
$$

Now there are two effects that influence $\gamma$, the direct effect through higher information revelation by the informed (overconfident) agents, plus the change in the fraction of informed agents. It can be easily verified from (8) that the product $\lambda_{o}^{*} b_{\epsilon}$ is increasing in $b_{\epsilon}$, therefore

\footnotetext{
${ }^{15}$ That is, when $m_{o} b_{\epsilon} \geq \tau \sigma_{\epsilon} \sigma_{z} \sqrt{C(\tau)^{-1}-\sigma_{\epsilon}^{2}}$.
} 
increasing information revelation. A higher value of $\gamma$ in turn implies that the impact of noise on the equilibrium price is reduced, and so are noise traders expected losses (and therefore other traders' expected profits and welfare). The effect of overconfidence on price volatility exhibits the same non-monotonic property discussed after Proposition 1, driven by the same forces.

\section{Extensions}

In this section of the paper we consider several models in which we illustrate the robustness of the previous results. We study more general information acquisition technologies, a version of the Grossman and Stiglitz (1980) model, and an imperfectly competitive market (as in Kyle (1985)).

\subsection{General information acquisition technologies}

Consider now the following variation of the basic model. Agents can obtain signals of the type $Y_{i}=X+\epsilon_{i}$, with $\epsilon_{i} \sim \mathcal{N}(0,1 / p)$. In order to obtain such signals traders need to pay the price, in units of the numeraire, equal to $c(p)$. We assume that $c(p) \geqslant 0, c^{\prime}(p)>0$ and $c^{\prime \prime}(p) \geq 0$, $\forall p>0$. Thus, the cost of their signal is increasing and convex in its precision. In this way we extend the basic model to allow for more general information gathering technologies. The overconfident, as before, erroneously believe to receive signals, after paying the $\operatorname{cost} c\left(p_{o}\right)$, with precision $b_{\epsilon} p_{o}$ for some $b_{\epsilon}>1$.

The competitive equilibrium in this variation of the model is defined as in section 2 . The equilibrium in information acquisition is characterized by fractions of informed agents $\lambda_{r}^{*}$ and $\lambda_{o}^{*}$, and precision levels $p_{r}^{*}$ and $p_{o}^{*}$, such that: (1) no uninformed agent would want to become informed; (2) no informed agent would be better off by choosing other precision levels $p \neq p^{*}$, or by becoming uninformed. ${ }^{16}$ The equilibrium in information acquisition follows Verrechia (1982), with the additional considerations that may arise if $\lambda_{r}^{*} \neq 1$. $^{17}$

For the purpose of characterizing the equilibrium, define the following function of the primitives:

$$
\Lambda^{*}=-\frac{m_{o} b_{\epsilon} p_{o}^{*}}{m_{r} p_{r}^{*}}+\frac{1}{m_{r} p_{r}^{*}} \sqrt{\frac{\tau \sigma_{z}^{2}\left(e^{-2 c\left(p_{r}^{*}\right) \tau}-2 \tau c^{\prime}\left(p_{r}^{*}\right)\right)}{c^{\prime}\left(p_{r}^{*}\right)}} ;
$$

where $p_{o}^{*}$ and $p_{r}^{*}$ are defined in the Appendix. The next Proposition describes the equilibrium

\footnotetext{
${ }^{16}$ Note that since in principle we do not exclude the case $c(0)>0$ we must allow for this possibility separately in the analysis.

${ }^{17}$ The assumptions in Verrechia (1982) imply that equation (29) in the Appendix never binds. In our symmetric model this means that either all agents become informed, or none does, as we show in the proof.
} 
in such economy.

Proposition 4 When traders can choose a signal of arbitrary precision, then the fraction of rational informed traders is given by: a) $\lambda_{r}^{*}=\Lambda^{*}$ if $\Lambda^{*} \in(0,1)$; b) $\lambda_{r}^{*}=1$ if $\Lambda^{*} \geq 1$; c) $\lambda_{r}^{*}=0$ if $\Lambda^{*} \leq 0$. The irrelevance result in Proposition 1 holds iff $\Lambda^{*} \in(0,1)$

If the parameters of the model are such that $\Lambda^{*} \in(0,1)$, then an interior fraction of rational agents becomes informed. The interpretation of $\Lambda^{*}$ as an irrelevance threshold is similar to the basic model: for $\Lambda^{*}$ to be positive it must be that

$$
m_{o} b_{\epsilon}<\frac{1}{p_{o}^{*}} \sqrt{\tau \sigma_{z}^{2}\left(e^{-2 c\left(p_{r}^{*}\right) \tau}-2 \tau c^{\prime}\left(p_{r}^{*}\right)\right) c^{\prime r}\left(p_{r}^{*}\right)^{-1}}
$$

where the lelf-hand side of the above expression can be interpreted as the degree of overconfidence, and the term on the right as some threshold level. The intuition of the irrelevance result goes back to the usual expression for the relative price coefficients $\gamma$, which in this case takes on the form

$$
\gamma=\frac{m_{o} b_{\epsilon} p_{o}^{*}}{\tau}+\frac{m_{r} \lambda_{r}^{*} p_{r}^{*}}{\tau}
$$

so that the impact of overconfidence is given by

$$
\frac{d \gamma}{d b_{\epsilon}}=\frac{m_{o} p_{o}^{*}}{\tau}+\frac{m_{o} b_{\epsilon}}{\tau} \frac{d p_{o}^{*}}{d b_{\epsilon}}+\frac{m_{r} p_{r}^{*}}{\tau} \frac{d \lambda_{r}^{*}}{d b_{\epsilon}}+\frac{m_{r} \lambda_{r}^{*}}{\tau} \frac{d p_{r}^{*}}{d b_{\epsilon}}
$$

The impact of overconfidence on price revelation is driven by the standard first two terms (more aggressive trading by the overconfident plus more information acquisition on their part), plus the two other terms which measure the response by rational agents to the higher levels of overconfidence. In the Appendix we show that when $\lambda_{r}^{*} \in(0,1)$, then rational traders react by scaling down the demand for information via the second term (response in the equilibrium fraction of informed traders) in a way that offsets the first two terms given by the increase of overconfidence, and the fourth term (response in the equilibrium precision) is equal to zero. On the other hand, if $\lambda_{r}^{*}=1$, then the third term is equal to zero and the offsetting effect comes from the fourth term, i.e. $d p_{r}^{*} / d b_{\epsilon}<0$, but is smaller in magnitude than the positive effect resulting from more aggressive trading by the uninformed, and therefore overconfidence will increase price informativeness.

\subsection{Correlated signals}

To inspect the robustness of our main result on overconfidence and informational efficiency, we consider the special case in which every informed agent gets a signal $Y_{i}=X+\epsilon_{i}$ with $\epsilon_{i}=\epsilon, \forall i$, i.e. the case where agents get signals whose errors are perfectly correlated. All other 
assumptions regarding the structure of the market are unchanged with respect to section 2 . This variation of the model is a direct extension of the model of Grossman and Stiglitz (1980), and allows us to argue that independence of the signals does not drive any of the results derive thus far. ${ }^{18}$ We define $\Lambda_{G S}^{*}$ as

$$
\Lambda_{G S}^{*}=\frac{1}{m_{r}}\left(\tau \sigma_{\epsilon} \sigma_{z} \sqrt{\frac{\left(1-C(\tau) \sigma_{\epsilon}^{2}\right)}{\left(1+\sigma_{\epsilon}^{2}\right) C(\tau)}}-m_{o} b_{\epsilon}\right)
$$

Again, we start by solving for the equilibrium price taking $\lambda_{o}, \lambda_{r}$ as exogenous and then we go back to the information acquisition stage and find the equilibrium values for $\lambda_{o}$ and $\lambda_{r}$.

Lemma 3 For given values of $\lambda_{j} \geq 0$, the competitive equilibrium price $P_{x}$ is given by $P_{x}=$ $\hat{a}\left(Y-\gamma^{-1} Z\right)$; where the coefficient $\hat{a}$ is a constant that depends on the primitives of the model, and

$$
\gamma=\frac{1}{\tau \sigma_{\epsilon}^{2}}\left(\lambda_{o} m_{o} b_{\epsilon}+\lambda_{r} m_{r}\right)
$$

As is customary in this setup, the price only transmits but does not aggregate the information, and the noise of the signal appears in the equilibrium price. Notice that in this model $\gamma$ is the relevant parameter for market efficiency, since

$$
\operatorname{var}\left(X \mid P_{x}\right)=\frac{\sigma_{\epsilon}^{2}+\frac{\sigma_{z}^{2}}{\gamma^{2}}}{1+\sigma_{\epsilon}^{2}+\frac{\sigma_{z}^{2}}{\gamma^{2}}}
$$

and that (14) is monotonically decreasing in $\gamma$, therefore implying that when $\lambda_{j}$ are exogenous, an increase in overconfidence, $b_{\epsilon}$ raises the amount of information revealed by the price.

The next Lemma characterizes the equilibrium with information acquisition.

Lemma 4 The equilibrium with information acquisition belongs to one of the following two classes:

(a) If the parameters of the model are such that $\Lambda_{G S}^{*}>0$, a fraction of the rational agents and all overconfident agents become informed, i.e. $\lambda_{o}^{*}=1$ and $\lambda_{r}^{*}=\Lambda_{G S}^{*}$.

(b) If the parameters of the model are such that $\Lambda_{G S}^{*}<0$, a fraction of the overconfident

\footnotetext{
${ }^{18}$ One can show that the irrelevance result holds for imperfectly correlated signals, i.e. signal structures of the form $Y_{i}=X+\epsilon+\epsilon_{i}$, where $\epsilon$ denotes a common error term, and the $\epsilon_{i}$ 's are i.i.d., which subsumes the model in section 2 and the one currently being discussed.
} 
traders becomes informed, but none of the rational agents, i.e. $\lambda_{r}^{*}=0$ and

$$
\lambda_{o}^{*}=\frac{\tau \sigma_{z}}{m_{o}} \sqrt{\frac{k_{\epsilon} \sigma_{\epsilon}^{2}\left(1-C(\tau) k_{\epsilon} \sigma_{\epsilon}^{2}\right)}{\left(1+k_{\epsilon} \sigma_{\epsilon}^{2}\right) C(\tau)}}
$$

The equilibrium with endogenous information acquisition shares the same properties of the basic model: rational traders will become informed only if all overconfident are informed. The following Proposition states an equivalent result to Proposition 1 for this variation of the basic model.

Proposition 5 If $\Lambda_{G S}^{*}>0$ then overconfidence is irrelevant for informational efficiency, that is, $\gamma$ is equal to what would endogenously arise in a fully rational economy.

The intuition for the result is identical: the rational traders, when they are the marginal buyers of information, scale back their information acquisition activities (less of them become informed), and this exactly offsets the standard effect of higher price informativeness stemming from more overconfidence. This shows that the result on the irrelevance of overconfidence for market efficiency is robust to other types of information structure in the market. It should be remarked that other variables of interest, and in particular the price function itself, do depend on the level of overconfidence $b_{\epsilon}$, in contrast to the case studied in section 3 . This dependence goes much along the same lines as in Odean (1998) (Model III) and will not be reported here for brevity.

\subsection{An imperfectly competitive model}

In order to further analyze the effects of overconfidence in markets populated by both rational and overconfident agents we now turn to study a multi-agent version of the Kyle (1985) model. The main departure point from the previous section is the fact that all agents are "large", in the sense that their trades affect prices. ${ }^{19}$ We recall that Odean (1998) and Benos (1998) showed that the introduction of overconfidence increases market depth. ${ }^{20}$ We show below that this result depends critically on the fact that informed agents are overconfident: once we allow for rational traders and endogeneous information acquisition a higher degree of overconfidence can make some rational agents drop out of the market, thereby decreasing market liquidity.

We consider a finite-agent economy, where all traders observe a signal of the form $Y_{i}=$ $X+\epsilon_{i}$, where $X \sim \mathcal{N}(0,1)$ denotes the final payoff of the risky asset, and $\epsilon_{i} \sim \mathcal{N}\left(0, \sigma_{\epsilon}^{2}\right)$. For

\footnotetext{
${ }^{19}$ The non-competitive assumption of the model to be discussed in this section does not play a role, as will become apparent in the following discussion. In particular, the same type of results could be developed in a finite-agent version of the Hellwig (1980) model where agents act as price takers.

${ }^{20}$ The analysis is also similar to Kyle and Wang (1997), although the emphasis in that paper is on the commitment benefits of overconfidence.
} 
simplicity all signals' errors $\epsilon_{i}$ are assumed to be independent. ${ }^{21}$ There are $m$ overconfident agents, who erroneously believe that the variance of their signal's estimation error is actually $k_{\epsilon} \sigma_{\epsilon}^{2}$, where $k_{\epsilon}<1$. We again let $b_{\epsilon}=1 / k_{\epsilon}$. In addition to overconfident agents, $n$ rational traders exist in the economy. These agents estimate the precision of their private signal correctly. In order to abstract from risk-aversion effects we let both overconfident and rational traders be expected profits maximizers. On top of these two types of agents, there are also noise traders in the market, who submit orders that we denote by $U$, where $U \sim \mathcal{N}\left(0, \sigma_{u}^{2}\right)$.

As usual in this type of models, prices are set by a risk-neutral market maker, who is assumed to be competitive (i.e. earn zero expected profits in equilibrium). Namely, the market maker sets prices conditional on total order flow. We let $\theta_{i}$ denote the trading strategy of agent $i$. All traders and the market maker are assumed to know the structure of the market, in particular they rationally anticipate the trading strategies of other types of traders, given their exogenously specified biases. ${ }^{22}$ The following definition formalizes the notion of an equilibrium in this type of model.

Definition 4 An equilibrium in the economy is defined by a set of trading strategies $\theta_{i}$ and a price function $P_{x}: \Omega \rightarrow \mathbb{R}$ such that:

1. Each agent $i$ chooses her trading strategy so as to maximize her expected profits given her signal $Y_{i}$ :

$$
\theta_{i} \in \arg \max _{\theta} \pi_{i}=\mathbb{E}^{i}\left[\theta_{i}\left(X-P_{x}\right) \mid Y_{i}\right]
$$

where if agent $i$ is overconfident the expectation is taken under the beliefs that $\epsilon_{i} \sim$ $\mathcal{N}\left(0, k_{\epsilon} \sigma_{\epsilon}^{2}\right)$, whereas if agent $i$ is rational $\epsilon_{i} \sim \mathcal{N}\left(0, \sigma_{\epsilon}^{2}\right)$.

2. The market maker breaks even:

$$
P_{x}=\mathbb{E}[X \mid \omega]
$$

where $\omega$ denotes the total order flow, i.e. $\omega=\sum_{i=1}^{n+m} \theta_{i}+U$.

The following lemma characterizes the equilibrium price and trading strategies. It essentially extends Benos (1998) to the case where agents have a varying degree of overconfidence.

Lemma 5 The equilibrium price and trading strategies are linear in $\omega$ and $Y_{i}$ respectively, i.e. price is given by $P_{x}=\lambda \omega$, rational agents' trading strategies are $\theta_{i}=\beta_{r} Y_{i}$ and those of the

\footnotetext{
${ }^{21}$ The fact that the signal precisions of all agents are the same plays no role in the analysis that follows (besides the notational simplicity).

${ }^{22}$ In principle the overconfident could commit errors in assessing the trading aggressiveness of the rational agents. Also, the market maker could have her own behavioral biases. We abstract from these in what follows and simply focus on overconfidence biases with respect to one's own signal.
} 
overconfident are $\theta_{i}=\beta_{o} Y_{i}$, where

$$
\begin{gathered}
\beta_{r}=\frac{\eta}{1+2 \sigma_{\epsilon}^{2}} ; \quad \beta_{o}=\frac{\eta}{1+2 k_{\epsilon} \sigma_{\epsilon}^{2}} ; \\
\lambda^{-1}=\eta\left(1+\frac{n}{1+2 \sigma_{\epsilon}^{2}}+\frac{m}{1+2 k_{\epsilon} \sigma_{\epsilon}^{2}}\right) \\
\eta^{2}=\sigma_{u}^{2}\left(\frac{n\left(1+\sigma_{\epsilon}^{2}\right)}{\left(1+2 \sigma_{\epsilon}^{2}\right)^{2}}+\frac{m\left(1+\left(2 k_{\epsilon}-1\right) \sigma_{\epsilon}^{2}\right)}{\left(1+2 k_{\epsilon} \sigma_{\epsilon}^{2}\right)^{2}}\right)^{-1} .
\end{gathered}
$$

A necessary and sufficient condition for an equilibrium to exist is that (20) defines a positive real number. ${ }^{23}$

As expected, the overconfident agents trade more aggressively than the rational. This is simply due to the fact that these agents believe their information to be more precise than that of the rational. It should nonetheless be noted that the trading aggressiveness of the overconfident is no longer a simple function of their behavioral bias: it now depends, through the market maker price setting, on the market wide variable $\eta$, which is itself a non-monotonic function of the bias measure $b_{\epsilon}$. The following proposition is immediate.

Proposition 6 If the number of informed agents $m$ and $n$ are exogeneously fixed, then market depth is increasing in overconfidence.

The proposition highlights the robustness of the positive effect of overconfidence on market liquidity, when information is exogenously fixed, reported elsewhere in the literature (Odean (1998), Benos (1998)). Compared to a purely rational economy, financial markets with overconfident will exhibit higher market depth.

We now turn to study the incentives to acquire information by rational agents. In particular, we fix the number (and information) of the overconfident, and allow a large number of rational agents to purchase a signal of precision $1 / \sigma_{\epsilon}^{2}$ for a cost $c$. We let $n^{*}$ denote the largest $n^{*}$ such that $\pi_{r}\left(n^{*}\right) \geq c$, i.e. $n^{*}$ denotes the largest number of rational agents such that if $n^{*}$ of them is informed it is still profitable for them to acquire information. This is the natural outcome of a standard Nash equilibrium in information acquisition in this type of setting.

The following proposition shows that the same forces that were in action in the competitive models play a role in this version of the Kyle (1985) model for moderate levels of overconfidence.

Proposition 7 Given $m$, let $n^{*}$ be determined endogenously. For moderate levels of overconfidence, $n^{*}$ is weakly decreasing in overconfidence. As a result, market depth can decrease as a function of overconfidence.

\footnotetext{
${ }^{23}$ In the analysis that follows we will always assume this condition to be satisfied
} 
The result in Proposition 7 highlights the robustness of the main effect which drives the irrelevance result of previous sections: ${ }^{24}$ rational agents' incentives to gather information are reduced when overconfidence appears. As discussed in Benos (1998), an increase in overconfidence (given $m$ and $n$ ) has two opposite effects on the aggressiveness of rational traders: a market liquidity effect and a strategic substitution effect. The first one is related to the increase in market depth, which causes rational traders be more aggressive; the second is related with the increase in the aggressiveness of the overconfident, which leads rational traders to trade less. When overconfidence is not too severe the second effect dominates, reducing expected trading profits of rational traders. ${ }^{25}$ This can in turn force some of them to drop out of the market and reduce market depth. ${ }^{26}$ One can view this result in light of the benefits of overconfidence as a commitment device, discussed in Kyle and Wang (1997) and Benos (1998). Namely, if there is heterogeneity with respect to commitment power, those agents that lack commitment will have less incentives to invest in information, compared to the economy where all agents lack this commitment power. This in turn can make the market less liquid.

\section{Conclusion}

This paper considers a model in which rational traders coexist with overconfident ones. We have shown that endogenizing the information acquisition decision generates significant predictions with respect to models with exogenous information distribution. In particular, there exist economies in which the equilibrium price corresponds to what would endogenously arise in a rational expectations equilibrium. The rational agents react to the presence of overconfident agents by reducing their information acquisition activities, since the returns to informed trading are reduced when overconfident agents trade more aggressively and thereby reveal more of their information through prices. On the other hand, we show that trading volume is generically increasing in overconfidence, confirming previous findings in the literature. Our results yield further insights into the interaction of overconfidence, information acquisition and price revelation in financial markets.

\footnotetext{
${ }^{24}$ In the finite-agent economies, such an irrelevance result is impossible to obtain, due to the discreteness of the model.

${ }^{25}$ In particular, a sufficient condition for $n^{*}$ to be weakly decreasing in overconfidence is that $2 k_{\epsilon} \sigma_{\epsilon}^{2}>2 \sigma_{\epsilon}^{2}-1$, which is clearly satisfied as $k_{\epsilon} \rightarrow 1$ or when $2 \sigma_{\epsilon}^{2}-1<0$.

${ }^{26}$ Consider the following numerical example: $\sigma_{\epsilon}^{2}=1 / 5 ; \sigma_{u}^{2}=2 ; c=0.1 ; m=2$. One can easily verify that for $k_{\epsilon}=0.5$ the model implies $n^{*}=3$ and $\lambda^{-1} \approx 3.8$, while for $k_{\epsilon}=0.4$ the model implies $n^{*}=2$ and $\lambda^{-1} \approx 3.6$.
} 


\section{Appendix}

\section{Proof of Lemma 1.}

By standard techniques, it is straightforward to see that the average trade by the overconfident can be written as

$$
\Theta_{o}=m_{o} \lambda_{o} \frac{b_{\epsilon}}{\tau \sigma_{\epsilon}^{2}} X+\left(\lambda_{o} q_{o}+\left(1-\lambda_{o}\right) w_{o}\right) P_{x}
$$

for some constants $q_{o}$ and $w_{o}$.

Similarly the average trade by the rational agents is given by

$$
\Theta_{r}=m_{r} \lambda_{r} \frac{1}{\tau \sigma_{\epsilon}^{2}} X+\left(\lambda_{r} q_{r}+\left(1-\lambda_{r}\right) w_{r}\right) P_{x}
$$

for some constants $q_{r}$ and $w_{r}$.

Using the market clearing condition (2) we obtain two equilibrium conditions from which (4) and (5) follow.

\section{Proof of Lemma 2.}

An informed overconfident agent $t$ gets expected utility (see Admati and Pfleiderer (1987))

$$
\mathbb{E}^{o}\left[u\left(W_{t}\right)\right]=-\sqrt{\frac{\operatorname{var}^{o}\left(X \mid Y_{t}, P_{x}\right)}{\operatorname{var}^{o}\left(X-P_{x}\right)}} e^{\tau c}
$$

On the other hand, an informed rational $t$ agent has expected utility

$$
\mathbb{E}\left[u\left(W_{t}\right)\right]=-\sqrt{\frac{\operatorname{var}\left(X \mid Y_{t}, P_{x}\right)}{\operatorname{var}\left(X-P_{x}\right)}} e^{\tau c} .
$$

Since $\operatorname{var}^{o}\left(X \mid Y_{t}, P_{x}\right)<\operatorname{var}\left(X \mid Y_{t}, P_{x}\right)$, it is immediate that in order for a rational agent to purchase a signal, it is necessary that all overconfident agents are also informed, i.e. $\lambda_{r}>0$ implies $\lambda_{o}=1$.

Now consider the ex-ante information acquisition decision of a rational agent. From the above expressions we see that a rational agent will buy information if and only if

$$
-\operatorname{var}\left(X \mid Y_{t}, P_{x}\right)^{1 / 2} e^{\tau c} \geq-\operatorname{var}\left(X \mid P_{x}\right)^{1 / 2}
$$


which can be expressed more explicitly as

$$
\left(1+\frac{\gamma^{2}}{\sigma_{z}^{2}}\right) e^{\tau c} \leq\left(1+\frac{\gamma^{2}}{\sigma_{z}^{2}}+\frac{b_{\epsilon}}{\sigma_{\epsilon}^{2}}\right)
$$

If a positive fraction of the rational agents becomes informed the above inequality must hold with equality. Substituting (4) into (23), and using $\lambda_{o}=1$ we the expression for $\lambda_{r}^{*}$ in the Lemma.

Lastly, for parameter values such that $\Lambda^{*}<0$ the above argument shows that none of the informed agents would choose to be informed, so $\lambda_{r}^{*}=0$. Moreover, equating the expected utilities of the overconfident when informed and uninformed we arrive at the expression

$$
-\operatorname{var}^{o}\left(X \mid Y_{t}, P_{x}\right)^{1 / 2} e^{\tau c} \geq-\operatorname{var}^{o}\left(X \mid P_{x}\right)^{1 / 2}
$$

from which, using (4), the expression in the Lemma for $\lambda_{o}^{*}$ follows.

\section{Proof of Proposition 1.}

By direct computation, using the expression for $\gamma$ from (4), and the endogenous $\lambda_{r}^{*}=\Lambda^{*}$ in (3), we have that

$$
\frac{d \gamma}{d b_{\epsilon}}=\frac{1}{\tau \sigma_{\epsilon}^{2}}\left(m_{o}+m_{r} \frac{d \lambda_{r}^{*}}{d b_{\epsilon}}\right)=0
$$

Further note that the price coefficient $d$ only depends on $b_{\epsilon}$ through $\gamma$, see equation (5). Therefore the price function is independent of $b_{\epsilon}$. This completes the proof.

\section{Proof of Proposition 2.}

The measure of informed traders, $m_{o} \lambda_{o}^{*}+m_{r} \lambda_{r}^{*}$, is decreasing in overconfidence when $\Lambda^{*}>0$, since in this case $\lambda_{o}^{*}=1$ and from expression (3) we have that

$$
m_{o}+m_{r} \Lambda^{*}=m_{o}+\left(\tau \sigma_{\epsilon} \sigma_{z} \sqrt{C(\tau)^{-1}-\sigma_{\epsilon}^{2}}-m_{o} b_{\epsilon}\right)
$$

The above expression valued at $b_{\epsilon}=1$ corresponds to the measure of informed traders in a fully rational economy, and is decreasing in $b_{\epsilon}$.

Trading volume is measured in expected terms, as the number of shares that are expected to be traded in the market. Each trader's expected trading volume, $T_{i}$ from now on, is given by the expectation of the absolute value of his trading strategy, i.e. $T_{i}=\left|\theta_{i}\right|$. Expected trading volume is defined as $\int_{i} T_{i} d i$, where the index of integration runs through all agents (overconfident and rational). Numerical simulations ${ }^{27}$ show that $\mathbb{E}[T]$ is increasing in $b_{\epsilon}$ for many sets of

\footnotetext{
${ }^{27}$ Using the fact that if $x \sim N\left(0, \sigma^{2}\right)$, then $\mathbb{E}[|x|]=\sqrt{\frac{2 \sigma^{2}}{\pi}}$.
} 
parameters specifying the economy (perhaps for all sets, no numerical counterexample was found). We further define expected returns from agent $i$ 's trading as $\mathbb{E}\left[R_{i}\right]=\mathbb{E}\left[\theta_{i}\left(X-P_{x}\right)\right]$. A direct computation using the expressions from Lemma 1 and Lemma 2 yields the conclusion in the Proposition. This completes the proof.

\section{Proof of Proposition 3.}

The proof follows immediately by inspection of (8).

\section{Proof of Proposition 4.}

An informed rational agent will choose $p$ so as to maximize

$$
\mathbb{E}\left[u\left(W_{t}\right)\right]=-\sqrt{\frac{\operatorname{var}\left(X \mid Y_{t}, P_{x}\right)}{\operatorname{var}\left(X-P_{x}\right)}} e^{\tau c(p)}
$$

where

$$
\operatorname{var}\left(X \mid Y_{t}, P_{x}\right)=\left(1+\frac{\gamma^{2}}{\sigma_{z}^{2}}+p\right)^{-1}
$$

taking $\gamma$ and the parameters of the price function as given. The first-order condition of (25) with respect to $p$ yields

$$
2 \tau c^{\prime}\left(p_{r}^{*}\right)\left[1+\frac{\gamma^{2}}{\sigma_{z}^{2}}+p_{r}^{*}\right]=1
$$

Similarly, an informed overconfident agent will choose $p_{o}^{*}$ such that

$$
2 \tau c^{\prime}\left(p_{o}^{*}\right)\left[1+\frac{\gamma^{2}}{\sigma_{z}^{2}}+b_{\epsilon} p_{o}^{*}\right]=1
$$

It is straightforward to show that no rational agent will become informed unless all overconfident choose to do so. As in the main body of the text we focus then on the case where $\lambda_{o}^{*}=1$. Equating the expected utilities of a rational informed (25) and a rational uninformed agent we get

$$
e^{2 \tau c\left(p_{r}^{*}\right)}\left(1+\frac{\gamma^{2}}{\sigma_{z}^{2}}\right)=\left(1+\frac{\gamma^{2}}{\sigma_{z}^{2}}+p_{r}^{*}\right)
$$

where $\gamma$ is given by (10). Substituting (10) and (27) into (29) we get a quadratic equation for $\lambda_{r}$, whose unique non negative solution yields

$$
\lambda_{r}^{*}=\Lambda^{*} \equiv-\frac{m_{o} b_{\epsilon} p_{o}^{*}}{m_{r} p^{*}}+\frac{1}{m_{r} p_{r}^{*}} \sqrt{\frac{\tau \sigma_{z}^{2}\left(e^{-2 c\left(p_{r}^{*}\right) \tau}-2 \tau c^{\prime}\left(p_{r}^{*}\right)\right)}{c^{\prime}\left(p_{r}^{*}\right)}}
$$

The above function gives the equilibrium value for $\lambda_{r}^{*}=\Lambda^{*}$ as long as $\Lambda^{*} \in(0,1)$, otherwise 
the equilibrium $\lambda_{r}^{*}$ is characterized by corner solutions $\left(\lambda_{r}^{*}=0\right.$ if $\Lambda^{*} \leq 0$ and $\lambda_{r}^{*}=1$ if $\left.\Lambda^{*} \geq 1\right)$. Assume now that the parameters are such that $\Lambda^{*} \in(0,1)$ and therefore $\lambda_{r}^{*}=\Lambda^{*}$ : substituting (30) into (10) it is easy to see that (10) is not a direct function of $b_{\epsilon}$ since the first term of (10) cancels out with the first term in (30). Therefore $d \gamma / d b_{\epsilon}=0$ as long as $d p / d b_{\epsilon}=0$. The last condition can be verified by substituting (10) into (27): since $\gamma$ is not directly a function of $b_{\epsilon}$ then the first-order condition for $p$ is not a function of $b_{\epsilon}$ neither. This yields the result that if $\lambda_{r}^{*}=\Lambda^{*}$ then $d \gamma / d b_{\epsilon}=0$.

On the other hand, now suppose that $\lambda_{r}^{*}=1$, i.e the constraint does not bind and all rational agents find it optimal to become informed. Applying the implicit function theorem to (27) we have

$$
\frac{d p^{*}}{d b_{\epsilon}}=-\frac{m_{o}}{m_{r} \sigma_{\epsilon}^{2}}\left(\frac{4 c^{\prime}\left(p^{*}\right) \gamma / \sigma_{z}^{2}}{4 c^{\prime}\left(p^{*}\right) \gamma / \sigma_{z}^{2}+2 \tau c^{\prime}\left(p^{*}\right) / m_{r}+2 \tau c^{\prime \prime}\left(p^{*}\right)\left(\operatorname{var}\left(X \mid \mathcal{F}_{I}\right) m_{r}\right)^{-1}}\right)
$$

Given the assumption on the cost function, i.e. $c^{\prime}\left(p^{*}\right)>0$ and $c^{\prime \prime}\left(p^{*}\right) \geq 0$, the fraction in parenthesis in the above expression is less than 1 . Then, it can be easily checked by substituting (31) into (11) that in this case $d \gamma / d b_{\epsilon}>0$.

\section{Proof of Lemma 3.}

Price is conjectured to be of the form:

$$
P_{x}=\hat{a} g(Y, Z)
$$

where $g(Y, Z)=Y-\frac{Z}{\gamma}$ and $\gamma=\frac{m_{o} \lambda_{o}}{\tau k_{\epsilon} \sigma_{\epsilon}^{2}}+\frac{m_{r} \lambda_{r}}{\tau \sigma_{\epsilon}^{2}}$. We will refer to $g(Y, Z)$ as the compound signal of $Y$ and $Z$. To understand how $g(Y, Z)$ is obtained, consider the market clearing condition

$m_{o}\left(\lambda_{o} \frac{\mathbb{E}^{o}\left(X \mid Y, P_{x}\right)-P_{x}}{\tau \operatorname{var}^{o}\left(X \mid Y, P_{x}\right)}+\left(1-\lambda_{o}\right) \frac{\mathbb{E}^{o}\left(X \mid P_{x}\right)-P_{x}}{\tau \operatorname{var}^{o}\left(X \mid P_{x}\right)}\right)+m_{r}\left(\lambda_{r} \frac{\mathbb{E}\left(X \mid Y, P_{x}\right)-P_{x}}{\tau \operatorname{var}\left(X \mid Y, P_{x}\right)}+\left(1-\lambda_{r}\right) \frac{\mathbb{E}\left(X \mid P_{x}\right)-P_{x}}{\tau \operatorname{var}\left(X \mid P_{x}\right)}\right)=Z$

Substituting for the conditional expectations and variances and solving for the equilibrium coefficients yields the expressions in the paper.

\section{Proof of Lemma 4.}

The proof follows closely the proof of Lemma 2, simply by changing the price function to that given in Lemma 3, and using the appropriate conditional moments (in particular note that for the informed agents their signal $Y$ is now a sufficient statistic for $X$, i.e. they do not condition their trade on price).

\section{Proof of Proposition 5.}


From the equilibrium equation for $\gamma$ in Lemma 3 and Lemma 4 we have that for $\Lambda^{*}>0$

$$
\frac{d \gamma}{d b_{\epsilon}}=\frac{1}{\tau \sigma_{\epsilon}^{2}}\left(m_{o}+m_{r} \frac{d \lambda_{r}^{*}}{d b_{\epsilon}}\right)=\frac{1}{\tau \sigma_{\epsilon}^{2}}\left(m_{o}+m_{r} \frac{d \Lambda^{*}}{d b_{\epsilon}}\right)
$$

Given the expression for $\Lambda^{*}$ it is straightforward to see that (33) is equal to zero.

\section{Proof of Lemma 5.}

Each agent maximizes his expected trading profits, $\pi_{i}=\theta_{i} \mathbb{E}\left[\left(X-P_{x}\right)\right]$, i.e. for the rational agents

$$
\max _{\theta_{i}} \quad \theta_{i} \mathbb{E}\left(X \mid Y_{i}\right)-\lambda \theta_{i}^{2}-\theta_{i} \lambda\left[(n-1) \beta_{r}+m \beta_{o}\right] \mathbb{E}\left(X \mid Y_{i}\right)
$$

which yields the optimal trading strategies

$$
\theta_{i}=\frac{\left(\lambda^{-1}-(n-1) \beta_{r}-m \beta_{o}\right)}{2\left(1+\sigma_{\epsilon}^{2}\right)} Y_{i} \equiv \beta_{r} Y_{i}
$$

Similarly for the overconfident traders we have

$$
\theta_{i}=\frac{\left(\lambda^{-1}-n \beta_{r}-(m-1) \beta_{o}\right)}{2\left(1+k_{\epsilon} \sigma_{\epsilon}^{2}\right)} Y_{i} \equiv \beta_{o} Y_{i}
$$

Some simple manipulations of (34) and (35) yields (18) for some constant $\eta$ that satisfies

$$
\eta+n \beta_{r}+m \beta_{o}=\lambda^{-1}
$$

It is straightforward to see, given the standard properties of normally distributed random variables, that $\mathbb{E}[X \mid \omega]=\lambda \omega$, where

$$
\lambda=\frac{n \beta_{r}+m \beta_{o}}{\left(n \beta_{r}+m \beta_{o}\right)^{2}+\left(n \beta_{r}^{2}+m \beta_{o}^{2}\right) \sigma_{\epsilon}^{2}+\sigma_{u}^{2}} .
$$

Using (37) with (36), (34) and (35) yields the expression for the equilibrium value for $\lambda$, namely equation (19).

\section{Proof of Proposition 6.}

If we let $\lambda^{-1}\left(k_{\epsilon}\right)$ denote the market depth as a function of the overconfidence bias, we have that $\lambda^{-1}(1)<\lambda^{-1}\left(k_{\epsilon}\right)$, for all $k_{\epsilon}<1 .^{28}$. The result directly follows from partially differentiating (19) with respect to $k_{\epsilon}$. Taking into account the condition for the existence of the equilibrium, it is easy to verify that $d \lambda^{-1} / d k_{\epsilon}<0$.

\footnotetext{
${ }^{28}$ This generalizes Benos (1998), who showed $\lambda^{-1}(1)<\lambda^{-1}(0)$.
} 


\section{Proof of Proposition 7.}

It is straightforward to compute the expected trading profits at equilibrium, $\pi_{r}=\mathbb{E}\left[\theta_{i}\left(X-P_{x}\right)\right]$, by the informed rational agents, which are given by

$$
\pi_{r}=\frac{\left(1+\sigma_{\epsilon}^{2}\right)}{\left(1+2 \sigma_{\epsilon}^{2}\right)^{2}} \eta \xi
$$

where

$$
\xi=\left(1+\frac{n}{1+2 \sigma_{\epsilon}^{2}}+\frac{m}{1+2 k_{\epsilon} \sigma_{\epsilon}^{2}}\right)^{-1}
$$

Therefore we have

$$
\frac{d \pi_{r}}{d k_{\epsilon}}=\frac{\left(1+\sigma_{\epsilon}^{2}\right)}{\left(1+2 \sigma_{\epsilon}^{2}\right)^{2}}\left(\frac{d \eta}{d k_{\epsilon}} \xi+\eta \frac{d \xi}{d k_{\epsilon}}\right)
$$

It is easy to verify that

$$
\operatorname{sign}\left(\frac{d \eta}{d k_{\epsilon}}\right)=\operatorname{sign}\left(-1+2 \sigma_{\epsilon}^{2}\left(1-k_{\epsilon}\right)\right)
$$

and that $d \xi / d k_{\epsilon}>0$. It follows that for $d \pi_{r} / d k_{\epsilon}>0$ a sufficient condition is $2 k_{\epsilon} \sigma_{\epsilon}^{2}>2 \sigma_{\epsilon}^{2}-1$. The second result follows immediately by considering small changes in the overconfidence parameter when the constraint $\pi_{r}\left(n^{*}\right) \geq c$ binds. 


\section{References}

Admati, A. R., and P. Pfleiderer, 1987, "Viable allocations of information in financial markets," Journal of Economic Theory, 43, 76-115.

Barber, B., and T. Odean, 2001, "Boys will be boys: gender, overconfidence and common stock investment," Quarterly Journal of Economics, 116, 261-292.

Barlevy, G., and P. Veronesi, 2000, "Information acquisition in financial markets," Review of Economic Studies, January.

Benos, A., 1998, "Aggressiveness and survival of overconfident traders," Journal of Financial Markets, 1, 353-383.

Bernardo, A., and I. Welch, 2001, "On the evolution of overconfidence and entrepreneurs," Cowles Foundation Working Paper.

Caballé, J., and J. Sàkovics, 2003, "Speculating against an overconfident market," Journal of Financial Markets, 6(2), 199-225.

Daniel, K., D. Hirshleifer, and A. Subrahmanyam, 1998, "A theory of overconfidence, self attribution, and security market under- and over-reaction," Journal of Finance, 53, 18391885.

— , 2001, "Overconfidence, arbitrage, and equilibrium asset pricing," Journal of Finance, $53,921-965$.

DeLong, J., A. Shleifer, L. Summers, and R. Waldmann, 1991, "The survival of noise traders in financial markets," Journal of Business, 64, 1-19.

Diamond, D. W., 1985, "Optimal release of information by firms," Journal of Finance, 40, 1071-1094.

Gervais, S., and T. Odean, 2001, "Learning to be overconfident," Review of Financial Studies, $14,1-27$.

Glaser, M., and M. Weber, 2003, "Overconfidence and trading volume," working paper, CEPR Working Paper.

Grossman, S., and J. E. Stiglitz, 1980, "On the impossibility of informationally efficient markets," American Economic Review, 70, 393-408.

Hellwig, M. F., 1980, "On the aggregation of information in competitive markets," Journal of Economic Theory, 22, 477-498. 
Kyle, A. S., 1985, "Continuous auctions and insider trading," Econometrica, 53, 1315-1336.

Kyle, A. S., and F. A. Wang, 1997, "Speculation duopoly with agreement to disagree: can overconfidence survive the market test?," Journal of Finance, 52, 2073-2090.

Long, J. D., A. Shleifer, L. Summers, and R. Waldmann, 1990, "Noise trader risk in financial markets," Journal of Political Economy, 98, 703-738.

Morrison, A., 2004, "Competition and information production in market maker models," Journal of Business.

Odean, T., 1998, "Volume, volatility, price and profit when all traders are above average," Journal of Finance, 53, 1887-1934.

Rubinstein, M., 2001, "Rational markets: yes or no? The affirmative case," Financial Analysts Journal.

Scheinkman, J., and W. Xiong, 2004, "Overconfidence and speculative bubbles," Journal of Political Economy.

Shleifer, A., 2000, "Inefficient markets: an introduction to behavioral finance," Oxford University Press.

Shleifer, A., and R. Vishny, 1997, "The limits of arbitrage," Journal of Finance, 52, 35-55.

Statman, M., S. Thorley, and K. Vorkink, 2003, "Investor overconfidence and trading volume," working paper, Santa Clara University.

Thaler, R., 1992, The winner's curse. Princeton University Press, Princeton, NJ.

— 1993, Advances in behavioral finance. Russell Sage Foundation, New York, NY.

Verrechia, R. E., 1982, "Information acquisition in a noisy rational expectations economy," Econometrica, 50, 1415-1430. 